\title{
XX Male
}

National Cancer Institute

\section{Source}

National Cancer Institute. XX Male. NCI Thesaurus. Code C86032.

A person who has a chromosome composition of $46 \mathrm{XX}$ and who is phenotypically male. 